\title{
Elevated mazes as animal models of anxiety: effects of serotonergic agents
}

\author{
SIMONE H. PINHEIRO ${ }^{1}$, HÉLIO ZANGROSSI-Jr. ${ }^{2}$, CRISTINA M. DEL-BEN ${ }^{1}$ \\ and FREDERICO G. GRAEFF ${ }^{1}$ \\ ${ }^{1}$ Departamento de Neurologia, Psiquiatria e Psicologia Médica, Hospital das Clínicas \\ Faculdade de Medicina de Ribeirão Preto, Universidade de São Paulo \\ Avenida Bandeirantes 3.900, 14048-900 Ribeirão Preto, SP, Brasil \\ ${ }^{2}$ Departamento de Farmacologia, Faculdade de Medicina de Ribeirão Preto, Universidade de São Paulo \\ Avenida Bandeirantes 3.900, 14049-900 Ribeirão Preto, SP, Brasil \\ Manuscript received on March 23, 2006; accepted for publication on April 12, 2006; \\ contributed by FREDERICO G. GRAEFF*
}

\begin{abstract}
This article reviews reported results about the effects of drugs that act upon the serotonergic neurotransmission measured in three elevated mazes that are animal models of anxiety. A bibliographic search has been performed in MEDLINE using different combinations of the key words X-maze, plus-maze, T-maze, serotonin and 5-HT, present in the title and/or the abstract, with no time limit. From the obtained abstracts, several publications were excluded on the basis of the following criteria: review articles that did not report original results, species other than the rat, intracerebral drug administration alone, genetically manipulated rats, and animals having any kind of experimental pathology. The reported results indicate that the effect of drugs on the inhibitory avoidance task performed in the elevated T-maze and on the spatio temporal indexes of anxiety measured in the $\mathrm{X}$ and plus mazes correlate with their effect in patients diagnosed with generalized anxiety disorder. In contrast, the drug effects on the one-way escape task in the elevated T-maze predict the drug response of panic disorder patients. Overall, the drug effects assessed with the avoidance task in the T-maze are more consistent than those measured through the anxiety indexes of the $\mathrm{X}$ and plus mazes. Therefore, the elevated T-maze is a promising animal model of generalized anxiety and panic disorder.
\end{abstract}

Key words: animal model, elevated maze, anxiety, panic, serotonin.

\section{INTRODUCTION}

Early animal models of anxiety have been developed on the basis of paradigms taken from the experimental psychology of the 1950s and 60s, before the modern classification of psychiatric disorders had split anxiety disorders in different diagnostic categories. As a consequence, these animal models refer to normal and pathological anxiety, in general. On the whole, they rely on either inhibition of ongoing behavior elicited by conditioned stimuli that predict unavoidable electric shock or on suppression of rewarded behavior by concurrent electric-shock

\footnotetext{
*Member Academia Brasileira de Ciências

Correspondence to: Prof. Frederico G. Graef

E-mail: fgraeff@keynet.com.br
}

punishment. The first paradigm is based on associative or Pavlovian conditioning, comprising the so-called conditional emotional response (CER) and the conditioned suppression of lever pressing (Estes and Skinner 1941, Millenson and Leslie 1974). The second, is based on instrumental or operant conditioning, and involves an approach-avoidance conflict. For this reason, the latter models have been called punishment or conflict tests.

Early pharmacological analysis has shown that conflict tests predict clinical drug response better than conditioned suppression (Kelleher and Morse 1968). As a consequence, conflict tests have become widely used for assaying anti-anxiety agents, either for screening purposes or for studying their mechanisms of action. How- 
ever, with the discovery of new anxiolytics, such as buspirone and ritanserin, which act primarily on the neurotransmission mediated by serotonin $(5-\mathrm{HT})$, it became clear that the available conflict tests failed to consistently detect their anxiolytic properties (Handley et al. 1993, Griebel 1995). Therefore, conflict tests seem to be fit only for detecting anxiolytics that act through the neurotransmission mediated by $\gamma$-aminobutyric acid (GABA), such as barbiturates or benzodiazepine agonists. Punishment tests have also been criticized because of their artificiality and the confounding influence of appetitive drives, such as hunger and thirst, as well as of pain (Treit 1985).

As these developments were taking place in basic research, classifications of anxiety disorders based on symptom clusters, time course and therapeutic response have been elaborated. Nowadays, the most widely used is part of the DSM IV classification of psychiatric disorders (American Psychiatric Association 1994), comprising the following primary anxiety disorders:

1. Generalized anxiety disorder (GAD), a state of excessive anxiety or apprehension lasting for more than six months. Neurovegetative symptoms are often present, but are relatively minor.

2. Panic disorder (PD), characterized by recurrent panic attacks, either unexpected or associated with particular situations. Panic attacks are sudden surges of intense fear or terror, desire of fleeing and feeling of imminent death, going crazy or loosing control. These subjective symptoms are accompanied by major neurovegetative changes, such as palpitation, hypertension, difficulty in breathing, sweating, urge to void the bladder and increased peristalsis. This leads to worry about the next attack or anticipatory anxiety, and avoidance of places where a panic attack would be embarrassing. Ultimately, generalized avoidance or agoraphobia may ensue. Nevertheless, agoraphobia sometimes occurs without panic attacks.

3. Obsessive-compulsive disorder characterized by intrusive, distressing thoughts (obsessions) and/or stereotyped or ritualized behavior (compulsions) that must be performed in order to alleviate intense anxiety.
4. Specific phobias, which are irrational fears of either objects (animals, blood, pointed instruments) or situations (heights, closed environments).

5. Social phobia (also called social anxiety disorder), or marked anxiety experienced in social situations, such as speaking in public, going to parties or being in a classroom.

The following discussion will refer to the first two categories, GAD and PD. The distinction between GAD and PD stemmed from the original observation by the North-American psychiatrist Donald Klein (Klein 1964) that chronic administration of the antidepressant imipramine improved PD, which was resistant to benzodiazepine anxiolytics at the dose regimens that improve GAD. This pharmacological distinction between the two categories has been further qualified, since it has been shown that chronic imipramine also improves GAD (Kahn et al. 1986) and high-potency benzodiazepines, such as alpralozam, are effective on PD when chronically administered (Schweizer et al. 1993). In spite of this, Donald Klein's postulation spurred research that revealed clear differences in the neurobiology of GAD and PD (for a recent review, see Ninan and Dunlop 2005).

The verification that each anxiety disorder engages a particular set of neurobiological systems led to the conclusion that animal models should address anxiety disorders specifically. Therefore, a given model is expected to have a pharmacological profile that correlates with the clinical drug response of the disorder it is intended to represent. Among the most important models of anxiety disorders that have been developed to replace the classical punishment tests are several types of elevated mazes, three of which are analyzed in the present article.

\section{ELEVATED MAZES}

The most widely used animal models of anxiety in the last two decades have been the elevated " $X$ " and "plus" mazes. Both the elevated X-maze (Handley and Mithani 1984) and the elevated plus-maze (Pellow et al. 1985) consist of two opposed arms enclosed by walls except at the central end. These closed arms are perpendicular to two open arms of equal dimensions, which are devoid of any wall. The whole apparatus is elevated above the 
floor. Since rats have innate fear of elevated open places, they enter less and stay for a shorter time in the open arms as compared to the closed arms when allowed to freely explore the maze. Typically, benzodiazepine anxiolytics increase the number of entries into and the time spent on the open arms. Countless drugs have been assayed in this model, and the results obtained summarized in several comprehensive reviews (e.g., Handley et al. 1993, Griebel 1995). In order to improve the capacity of the elevated plus-maze for detecting non-benzodiazepine anxiolytics, ethological analysis of behavioral items shown by the animals while exploring the elevated plus-maze (e.g., nose poking out of the enclosed arm and exploration of the open arm end) has been added to the former, spatio temporal measures (Cruz et al. 1994, Rodgers and Johnson 1995). However, this modification takes away one of the main advantages of the test, which is simplicity.

The British psychologist Sheila Handley, who conceived the idea of the crossed elevated maze, has argued that many of the inconsistencies found with drugs that alter 5-HT neurotransmission in the elevated $X$ and plus mazes may be explained by the fact that these are mixed models, in the sense that the rat displays different strategies of defense while exploring them, which could be influenced in opposite directions by 5-HT (Handley et al. 1993). At least two strategies may be easily seen: 1) avoidance of open arms when the rat is in one of the closed arms, and 2) escape from an open arm to enter a safer, closed arm. This observation led to the idea of separating these behavioral tasks: inhibitory avoidance and one-way escape, a goal that has been achieved by building the elevated T-maze (Graeff et al. 1993).

The elevated T-maze results from shutting off the entrance to one of the closed arms of the elevated plusmaze. For the inhibitory avoidance task, the rat is placed at the end of the remaining closed arm and the latency to withdraw from this arm with the four paws is recorded in three successive trials made at 30 -s intervals. Learning is indicated by the increase of the withdrawal latency along the trials. For the escape task, which initiates $30 \mathrm{~s}$ after the completion of the avoidance training, the rat is placed at the end of one of the open arms and the withdrawal latency from this arm is similarly recorded. In the studies performed so far, the number of trials of this task has varied from one to three. Pre-exposure to the open arm for
$30 \mathrm{~min}, 24 \mathrm{~h}$ before the test has been found to decrease de first withdrawal latency from the open arm and increase the drug sensitivity of the escape task (Teixeira et al. 2000). The resemblance of the motor performance in both tasks serves as a control for non-specific drug effects on motor activity when the latencies to withdraw from the enclosed arm and from the open arm are changed to opposite directions by the drug treatment. However, whenever the latencies are similarly increased or decreased, there is need for independent assessment of motor drug effects. Measuring motor activity inside an arena fulfils this requirement, albeit adding more complexity to the test.

Behavioral and pharmacological validation of the elevated T-maze (Graeff et al. 1998, Zangrossi Jr and Graeff 1997) has shown that the increase in avoidance latency is a function of the aversive character of the open arm, and that the pharmacological profile of the avoidance task is similar to that of GAD. In contrast, the escape task is insensitive to doses of benzodiazepines that have an anxiolytic effect (decrease of withdrawal latency) in the avoidance task, and is impaired by chronic, but not acute administration of imipramine (Teixeira et al. 2000, Zanoveli et al. 2005), clomipramine and fluoxetine (Poltronieri et al. 2003), drugs that are used to treat PD. Moreover, avoidance has been shown to be enhanced by a CCK receptor agonist (Zanoveli et al. 2004), a drug class that has been shown to induce panic attack in PD patients (Graeff et al. 2005). As a result, one-way escape in the elevated T-maze may be viewed as an animal model of PD.

\section{OBJeCTIVE}

A critical issue is whether the introduction of the T-maze represents an improvement over the preceding $\mathrm{X}$ and plus mazes in terms of consistency and predictive value of pharmacological effects of drugs that act on 5-HTmediated neurotransmission. To address this question, we compare the results reported in the literature describing the effects of such drugs on the behavioral indexes of anxiety and panic measured in the three above types of elevated mazes. 


\section{MATERIALS AND METHODS}

A MEDLINE search has been made using different combinations of the key words X-maze, plus-maze, T-maze, serotonin and 5-HT present in the title and/or the abstract with no time limit. From the obtained abstracts, several publications were excluded on the basis of the following criteria: review articles that did not report original results, species other than the rat, intracerebral drug administration alone, genetically manipulated rats, and animals having any kind of experimental pathology. As a result, we selected 20 articles for reviewing, which are quoted in Tables I and II. Further references have been added for the sake of introduction and discussion.

\section{REPORTED RESULTS AND DISCUSSION}

To improve clarity of analysis, the reviewed results are divided into two sets; the first comprises results obtained with 5-HT reuptake inhibitors and the second, with drugs that act as agonists or antagonists on different subtypes of 5-HT receptors.

\section{5-HT REUPTAKE INHIBITORS}

Nowadays, 5-HT reuptake inhibitors are the most widely used class of drugs in the treatment of anxiety disorders. They are first-choice medication for both GAD and PD, in addition to obsessive-compulsive disorder and social anxiety disorder (Nutt 2005).

The reported results on 5-HT reuptake inhibitors that we found in the literature survey are summarized in Table I.

Meaningful clinical correlations have been found in regard to the effects of imipramine, clomipramine and paroxetine on the two tasks of the elevated T-maze. Both imipramine and clomipramine enhanced avoidance after single administration, and this anxiogenic effect has been related to the initial worsening that has been observed during the first week of medication with these drugs in PD patients (Gentil et al. 1993). On the other hand, the anxiolytic effect of imipramine on the same task following chronic administration has been correlated with amelioration of GAD, which has been observed following repeated administration of the same drug for several weeks (Kahn et al. 1986). More important, the impairment of escape in the elevated T-maze caused by these three drugs correlates with their antipanic effect docu- mented in clinical assays (Klein 1964, Gentil et al. 1993, Ballenger et al. 1998).

In contrast, not a single drug correlation has been found between the effect of these drugs on the special indexes of anxiety measured in the elevated plus-maze, although it is arguable that the contrast between the anxiogenic effect of single administration and the anxiolytic effect of chronic administration of cianopramine reported in the elevated plus maze (Griebel et al. 1994a) may correlate with the effect of this drug on GAD. Nevertheless, such clinical effects have not yet been reported.

The inconsistency of the effect of single administration of fluoxetine on the elevated plus maze is particularly remarkable: anxiolytic in one study (Griebel et al. 1999), anxiogenic in three (Silva et al. 1999, Silva and Brandão 2000) and unchanged in another one (Griebel et al. 1997). The same occurs following chronic administration: unchanged (Griebel et al. 1999, Silva and Brandão 2000), versus anxiogenic (Silva et al. 1999).

\section{5-HT RECEPTOR LIGANDS}

The reported results on the effects of agonist and antagonist agents act, more or less selectively, on different subtypes of 5-HT receptors are summarized in Table II.

Among the drugs listed in Table II, only buspirone has been used in clinical practice, and has been tested in the three types of elevated mazes. In the two documented studies performed in the elevated T-maze, single administration of buspirone had an anxiolytic effect in the avoidance task, while not affecting escape performance (Graeff et al. 1998, Poltronieri et al. 2003). This correlates with the beneficial effect of this drug reported in GAD patients (Pecknold 1997). Nevertheless, the clinical effect requires several weeks of repeated drug administration to become apparent. So far, the results with chronic administration of buspirone on the avoidance task in the T-maze are inconsistent, since both no change (Poltronieri et al. 2003) and impairment an anxiolytic effect (Zanoveli et al. 2005) have been reported. As clinical evidence shows that the drug fails to improve PD when given chronically (Pecknold 1997), the absence of effect on escape reported both by Poltronieri et al. (2003) and Zanoveli et al. (2005) may be viewed as a positive clinical correlation.

In only one out of three studies carried out in the 
TABLE I

Comparative effects of reuptake inhibitors on rat behavior in two elevated mazes.

\begin{tabular}{|c|c|c|c|c|c|}
\hline \multirow[b]{2}{*}{$\begin{array}{l}\text { Drug } \\
\text { Action }\end{array}$} & \multirow[b]{2}{*}{ Reference } & \multirow{2}{*}{$\begin{array}{l}\text { Administration } \\
\text { (mg/kg, route) }\end{array}$} & \multicolumn{2}{|c|}{ T-maze } & \multirow{2}{*}{$\begin{array}{c}\text { Plus-maze } \\
\text { Open-arm } \\
\text { entries/time }\end{array}$} \\
\hline & & & $\begin{array}{l}\text { Inhibitory } \\
\text { avoidance }\end{array}$ & $\begin{array}{c}\text { One-way } \\
\text { escape }\end{array}$ & \\
\hline \multirow[t]{5}{*}{$\begin{array}{l}\text { Imipramine } \\
N A>5-H T\end{array}$} & $\begin{array}{c}\text { Teixeira } \\
\text { et al. }(2000)\end{array}$ & $\begin{array}{c}\text { Single } \\
(5-15, \text { IP })\end{array}$ & $\begin{array}{c}\text { Enhanced } \\
\text { (Anxiogenic) }\end{array}$ & Unchanged & Not tested \\
\hline & & $\begin{array}{c}21 \text { days } \\
(5-15, \text { IP) }\end{array}$ & $\begin{array}{c}\text { Impaired } \\
\text { (Anxiolytic) }\end{array}$ & $\begin{array}{l}\text { Impaired } \\
\text { (Antipanic) }\end{array}$ & Not tested \\
\hline & $\begin{array}{c}\text { Zanoveli } \\
\text { et al. (2005) }\end{array}$ & $\begin{array}{c}3 \text { days } \\
(15, \mathrm{IP})\end{array}$ & Unchanged & Unchanged & Not tested \\
\hline & & $\begin{array}{l}21 \text { days } \\
(15, \mathrm{IP})\end{array}$ & $\begin{array}{c}\text { Impaired } \\
\text { (Anxiolytic) }\end{array}$ & $\begin{array}{l}\text { Impaired } \\
\text { (Antipanic) }\end{array}$ & Not tested \\
\hline & $\begin{array}{c}\text { Griebel } \\
\text { et al. (1997) }\end{array}$ & $\begin{array}{c}\text { Single } \\
(1-10, \mathrm{SC})\end{array}$ & Not tested & Not tested & Unchanged \\
\hline \multirow[t]{3}{*}{$\begin{array}{l}\text { Clomipramine } \\
5-H T>N A\end{array}$} & $\begin{array}{c}\text { Graeff } \\
\text { et al. (1998) }\end{array}$ & $\begin{array}{l}\text { Single } \\
(10, \mathrm{IP})\end{array}$ & $\begin{array}{c}\text { Enhanced } \\
\text { (Anxiogenic) }\end{array}$ & Unchanged & Not tested \\
\hline & $\begin{array}{l}\text { Poltronieri } \\
\text { et al. (2003) }\end{array}$ & $\begin{array}{c}\text { Single } \\
(3-30, \text { IP })\end{array}$ & Unchanged & Unchanged & Not tested \\
\hline & & $\begin{array}{c}21 \text { days } \\
(3-30, \text { IP })\end{array}$ & Unchanged & $\begin{array}{l}\text { Impaired } \\
\text { (Antipanic) }\end{array}$ & Not tested \\
\hline $\begin{array}{l}\text { Subutramine } \\
N A / 5-H T^{1}\end{array}$ & $\begin{array}{c}\text { Jorge } \\
\text { et al. (2004) }\end{array}$ & $\begin{array}{c}\text { Single } \\
(5-20, \text { IP })\end{array}$ & $\begin{array}{c}\text { Decrease } \\
\text { (Anxiolytic) }\end{array}$ & $\begin{array}{c}\text { Decrease } \\
\text { (Antipanic) }\end{array}$ & Unchanged \\
\hline \multirow[t]{7}{*}{$\begin{array}{l}\text { Fluoxetine } \\
5-H T^{1}\end{array}$} & $\begin{array}{l}\text { Poltronieri } \\
\text { et al. (2003) }\end{array}$ & $\begin{array}{c}\text { Single } \\
(5-15, \text { IP })\end{array}$ & Unchanged & Unchanged & Not tested \\
\hline & & $\begin{array}{c}21 \text { days } \\
(5-15, \mathrm{IP})\end{array}$ & Unchanged & $\begin{array}{l}\text { Impaired } \\
\text { (Antipanic) }\end{array}$ & Not tested \\
\hline & $\begin{array}{c}\text { Griebel } \\
\text { et al. (1997) }\end{array}$ & $\begin{array}{c}\text { Single } \\
(1-10, \mathrm{SC})\end{array}$ & Not tested & Not tested & Unchanged \\
\hline & $\begin{array}{c}\text { Griebel } \\
\text { et al. (1999) }\end{array}$ & $\begin{array}{l}\text { Single } \\
(20, \text { IP) }\end{array}$ & Not tested & Not tested & $\begin{array}{c}\text { Increased } \\
\text { (Anxiolytic) }\end{array}$ \\
\hline & & $\begin{array}{c}\text { 23-24 days } \\
(20, \mathrm{IP})\end{array}$ & Not tested & Not tested & Unchanged \\
\hline & $\begin{array}{c}\text { Silva } \\
\text { et al. (1999) }\end{array}$ & $\begin{array}{l}\text { Single } \\
(5, \mathrm{IP})\end{array}$ & Not tested & Not tested & $\begin{array}{c}\text { Decreased } \\
\text { (Anxiogenic) }\end{array}$ \\
\hline & & $\begin{array}{c}21 \text { days } \\
(5, \mathrm{IP})\end{array}$ & Not tested & Not tested & $\begin{array}{c}\text { Decreased } \\
\text { (Anxiogenic) }\end{array}$ \\
\hline
\end{tabular}


TABLE I (continuation)

\begin{tabular}{|c|c|c|c|c|c|}
\hline \multirow{2}{*}{$\begin{array}{l}\text { Drug } \\
\text { Action }\end{array}$} & \multirow[b]{2}{*}{ Reference } & \multirow{2}{*}{$\begin{array}{l}\text { Administration } \\
(\mathrm{mg} / \mathrm{kg}, \text { route })\end{array}$} & \multicolumn{2}{|c|}{ T-maze } & \multirow{2}{*}{$\begin{array}{c}\text { Plus-maze } \\
\text { Open-arm } \\
\text { entries/time }\end{array}$} \\
\hline & & & $\begin{array}{l}\text { Inhibitory } \\
\text { avoidance }\end{array}$ & $\begin{array}{l}\text { One-way } \\
\text { escape }\end{array}$ & \\
\hline \multirow[t]{2}{*}{$\begin{array}{l}\text { Fluoxetine } \\
\text { 5-HT }\end{array}$} & $\begin{array}{l}\text { Silva and } \\
\text { Brandão } \\
(2000)\end{array}$ & $\begin{array}{l}\text { Single } \\
(10, \mathrm{IP})\end{array}$ & Not tested & Not tested & $\begin{array}{c}\text { Decreased } \\
\text { (Anxiogenic) }\end{array}$ \\
\hline & & $\begin{array}{l}14 \text { days } \\
(10, \mathrm{IP})\end{array}$ & Not tested & Not tested & Unchanged \\
\hline \multirow[t]{3}{*}{$\begin{array}{l}\text { Paroxetine } \\
\text { 5-HT }\end{array}$} & $\begin{array}{c}\text { Beijamini and } \\
\text { Andreatini } \\
\text { (2003) }\end{array}$ & $\begin{array}{c}3 \text { times in } 24 \mathrm{~h} \\
(5, \mathrm{VO})\end{array}$ & $\begin{array}{c}\text { Impaired } \\
\text { (Anxiolytic) }\end{array}$ & Unchanged & Not tested \\
\hline & & $\begin{array}{c}7 \text { days } \\
(5, \mathrm{VO})\end{array}$ & $\begin{array}{l}\text { Impaired }^{3} \\
\text { (Anxiolytic) }\end{array}$ & $\begin{array}{l}\text { Impaired }^{3} \\
\text { (Antipanic) }\end{array}$ & Not tested \\
\hline & $\begin{array}{c}\text { Koks } \\
\text { et al. }(2001)\end{array}$ & $\begin{array}{l}\text { Single } \\
(0.5, \text { IP })\end{array}$ & Not tested & Not tested & $\begin{array}{c}\text { Decreased } \\
\text { (Anxiogenic) }\end{array}$ \\
\hline $\begin{array}{c}\text { Zimelidine } \\
\text { 5-HT }\end{array}$ & $\begin{array}{c}\text { Griebel } \\
\text { et al. (1997) }\end{array}$ & $\begin{array}{c}\text { Single } \\
(1-10, \mathrm{SC})\end{array}$ & Not tested & Not tested & Unchanged \\
\hline $\begin{array}{c}\text { Fluvoxamine } \\
\text { 5- } H T^{1}\end{array}$ & $\begin{array}{c}\text { Griebel } \\
\text { et al. (1997) }\end{array}$ & $\begin{array}{c}\text { Single } \\
(1-10, \mathrm{SC})\end{array}$ & Not tested & Not tested & Unchanged \\
\hline $\begin{array}{c}\text { Citalopram } \\
\text { 5-HT } \\
\end{array}$ & $\begin{array}{c}\text { Griebel } \\
\text { et al. (1994a) }\end{array}$ & $\begin{array}{c}\text { Single } \\
(10,30, \text { IP })\end{array}$ & Not tested & Not tested & $\begin{array}{c}\text { Decreased } \\
\text { (Anxiogenic) }\end{array}$ \\
\hline $\begin{array}{c}\text { Cianopramine } \\
\text { 5- } H T^{1}\end{array}$ & $\begin{array}{c}\text { Griebel } \\
\text { et al. (1994a) }\end{array}$ & $\begin{array}{c}\text { Single } \\
(10, \mathrm{SC}) \\
21 \text { days } \\
(10, \mathrm{SC})\end{array}$ & $\begin{array}{l}\text { Not tested } \\
\text { Not tested }\end{array}$ & $\begin{array}{l}\text { Not tested } \\
\text { Not tested }\end{array}$ & $\begin{array}{c}\text { Decreased } \\
\text { (Anxiogenic) } \\
\text { Increased } \\
\text { (Anxiolytic) }\end{array}$ \\
\hline $\begin{array}{c}\text { Fenfluramine } \\
\text { 5-HT }\end{array}$ & $\begin{array}{c}\text { Graeff } \\
\text { et al. (1996) } \\
\end{array}$ & $\begin{array}{c}\text { Single } \\
(0.03-0.3, \text { IP }) \\
\end{array}$ & Enhanced $^{4}$ & $\begin{array}{c}\text { Impaired } \\
\text { (Antipanic) }\end{array}$ & Not tested \\
\hline
\end{tabular}

5-HT: serotonin; NA: noradrenaline; IP: intraperitoneal; SC: subcutaneous; VO: oral. Bold character words indicate known clinical correlation. ${ }^{1}$ Selective agent, not affecting neurotransmitter receptors; ${ }^{2}$ also a 5 -HT releaser; ${ }^{3}$ clinical improvement only after several weeks; ${ }^{4}$ nearly significant trend.

elevated plus-maze, buspirone had an anxiolytic effect (Griebel et al. 1997). In the remaining two (Moser et al. 1990, Collinson and Dawson 1997) as well as in the only study reported in the elevated X-maze (Critchley et al. 1992), an anxiogenic effect has been observed. Since chronic administration has not been explored, clinical correlation cannot be appropriately discussed.

Although the buspirone analog ipsapirone has not been marketed, experimental clinical trials have demonstrated its efficacy in GAD patients (Cutler et al. 1993). Like buspirone, ipsapirone had an anxiolytic effect in the avoidance task in the elevated T-maze after single administration (Graeff et al. 1998). An anxiolytic effect of the same drug has also been documented in the X-maze (Critchley et al. 1992), but not in the plus-maze (Setem et al. 1999).

Experimental clinical assays have been carried out with gepirone, another buspirone analog, showing a therapeutic action in GAD patients (Rickels et al. 1997). So far, this drug has not been tested in the elevated T-maze, but interesting results have been reported in the elevated plus maze, namely an anxiogenic effect after single ad- 
TABLE II

Comparative effects of 5-HT receptor ligands on rat behavior in two elevated mazes.

\begin{tabular}{|c|c|c|c|c|c|}
\hline \multirow{2}{*}{$\begin{array}{l}\text { Drug } \\
\text { Action }\end{array}$} & \multirow[b]{2}{*}{ Reference } & \multirow{2}{*}{$\begin{array}{l}\text { Administration } \\
\text { (mg/kg, route) }\end{array}$} & \multicolumn{2}{|c|}{ T-maze } & \multirow{2}{*}{$\begin{array}{c}\text { Plus-maze } \\
\text { Open-arm } \\
\text { entries/time }\end{array}$} \\
\hline & & & $\begin{array}{l}\text { Inhibitory } \\
\text { avoidance }\end{array}$ & $\begin{array}{l}\text { One-way } \\
\text { escape }\end{array}$ & \\
\hline $\begin{array}{c}\text { 5-MeODMT } \\
\text { 5-HT } 1 / 2 \\
\text { agonist } \\
\end{array}$ & $\begin{array}{c}\text { Critchley and } \\
\text { Handley }(1987)^{2}\end{array}$ & $\begin{array}{c}\text { Single } \\
(0.25-2.5, \mathrm{IP})\end{array}$ & Not tested & Not tested & $\begin{array}{c}\text { Decreased } \\
\text { (Anxiogenic) }\end{array}$ \\
\hline $\begin{array}{c}\text { RU } 24969 \\
\text { 5-HT } 1 / 2 \\
\text { agonist }\end{array}$ & $\begin{array}{c}\text { Critchley and } \\
\text { Handley }(1987)^{2} \\
\text { Critchley } \\
\text { et al. }(1992)^{2}\end{array}$ & $\begin{array}{c}\text { Single } \\
(0.1-3, \text { IP }) \\
\text { Single } \\
(0.5-2, \text { IP })\end{array}$ & Not tested & Not tested & $\begin{array}{c}\text { Decreased } \\
\text { (Anxiogenic) } \\
\text { Decreased } \\
\text { (Anxiogenic) }\end{array}$ \\
\hline \multirow[t]{6}{*}{$\begin{array}{c}\text { 8-OH-DPAT } \\
\text { 5-HT } T_{1 A} \\
\text { agonist }\end{array}$} & $\begin{array}{c}\text { Critchley and } \\
\text { Handley }(1987)^{2}\end{array}$ & $\begin{array}{c}\text { Single } \\
(0.015-1, \text { IP) }\end{array}$ & Not tested & Not tested & $\begin{array}{c}\text { Decreased } \\
\text { (Anxiogenic) }\end{array}$ \\
\hline & $\begin{array}{c}\text { Moser et al. } \\
\text { (1990) }\end{array}$ & $\begin{array}{c}\text { Single } \\
(12.5-200, \mathrm{SC})\end{array}$ & Not tested & Not tested & $\begin{array}{c}\text { Decreased } \\
\text { (Anxiogenic) }\end{array}$ \\
\hline & $\begin{array}{c}\text { Critchley } \\
\text { et al. }(1992)^{2}\end{array}$ & $\begin{array}{c}\text { Single } \\
(0.05-0.2, \mathrm{IP})\end{array}$ & Not tested & Not tested & $\begin{array}{c}\text { Decreased } \\
\text { (Anxiogenic) }\end{array}$ \\
\hline & $\begin{array}{c}\text { McBlane and } \\
\text { Handley (1994) }\end{array}$ & $\begin{array}{c}\text { Single } \\
(0.1,0.2, \mathrm{IP})\end{array}$ & Not tested & Not tested & $\begin{array}{c}\text { Decreased }^{3} \\
\text { (Anxiogenic) }\end{array}$ \\
\hline & $\begin{array}{c}\text { Collinson and } \\
\text { Dawson (1997) }\end{array}$ & $\begin{array}{c}\text { Single } \\
(0.01-0.3, \mathrm{SC})\end{array}$ & Not tested & Not tested & $\begin{array}{c}\text { Increased } \\
\text { (Anxiolytic) }\end{array}$ \\
\hline & $\begin{array}{c}\text { Griebel } \\
\text { et al. (1997) }\end{array}$ & $\begin{array}{c}\text { Single } \\
(0.1-1, \mathrm{SC})\end{array}$ & Not tested & Not tested & Unchanged \\
\hline $\begin{array}{c}\text { BAY R } 1521 \\
\text { 5-H } T_{1 A} \\
\text { agonist }\end{array}$ & $\begin{array}{c}\text { Critchley } \\
\text { et al. }(1992)^{2}\end{array}$ & $\begin{array}{c}\text { Single } \\
(0.1-1.2, \text { IP })\end{array}$ & Not tested & Not tested & $\begin{array}{c}\text { Decreased } \\
\text { (Anxiogenic) }\end{array}$ \\
\hline $\begin{array}{c}\text { Flesinoxan } \\
\text { 5-HT } T_{1 A} \\
\text { agonist }\end{array}$ & $\begin{array}{c}\text { Griebel } \\
\text { et al. (1997) }\end{array}$ & $\begin{array}{c}\text { Single } \\
(0.1-1, \mathrm{SC})\end{array}$ & Not tested & Not tested & Unchanged \\
\hline \multirow[t]{2}{*}{$\begin{array}{c}\text { Buspirone } \\
\text { 5-HT } T_{1 A} \\
\text { partial } \\
\text { agonist }\end{array}$} & $\begin{array}{c}\text { Graeff } \\
\text { et al. (1998) }\end{array}$ & $\begin{array}{c}\text { Single } \\
(0.3, \mathrm{IP})\end{array}$ & $\begin{array}{l}\text { Impaired }^{1} \\
\text { (Anxiolytic) }\end{array}$ & Unchanged & Not tested \\
\hline & $\begin{array}{l}\text { Poltronieri } \\
\text { et al. (2003) }\end{array}$ & $\begin{array}{c}\text { Single } \\
(0.3-3, \text { IP })\end{array}$ & $\begin{array}{l}\text { Impaired }^{1} \\
\text { (Anxiolytic) }\end{array}$ & Unchanged & Not tested \\
\hline
\end{tabular}


TABLE II (continuation)

\begin{tabular}{|c|c|c|c|c|c|}
\hline \multirow[b]{2}{*}{$\begin{array}{l}\text { Drug } \\
\text { Action }\end{array}$} & \multirow[b]{2}{*}{ Reference } & \multirow[b]{2}{*}{$\begin{array}{l}\text { Administration } \\
\text { (mg/kg, route) }\end{array}$} & \multicolumn{2}{|c|}{ T-maze } & \multirow{2}{*}{$\begin{array}{c}\text { Plus-maze } \\
\text { Open-arm } \\
\text { entries/time }\end{array}$} \\
\hline & & & $\begin{array}{l}\text { Inhibitory } \\
\text { avoidance }\end{array}$ & $\begin{array}{l}\text { One-way } \\
\text { escape }\end{array}$ & \\
\hline \multirow{6}{*}{$\begin{array}{c}\text { Buspirone } \\
5-H T_{1 A} \\
\text { partial } \\
\text { agonist }\end{array}$} & $\begin{array}{l}\text { Poltronieri } \\
\text { et al. (2003) }\end{array}$ & $\begin{array}{c}21 \text { days } \\
(0.3-3, \mathrm{IP})\end{array}$ & Unchanged & Unchanged & Not tested \\
\hline & $\begin{array}{c}\text { Zanoveli } \\
\text { et al. (2005) }\end{array}$ & $\begin{array}{l}21 \text { days } \\
(0.3, \mathrm{IP})\end{array}$ & $\begin{array}{c}\text { Impaired } \\
\text { (Anxiolytic) }\end{array}$ & Unchanged & Not tested \\
\hline & $\begin{array}{c}\text { Moser et al. } \\
\text { (1990) }\end{array}$ & $\begin{array}{c}\text { Single } \\
(0.5-2, \mathrm{SC})\end{array}$ & Not tested & Not tested & $\begin{array}{c}\text { Decreased } \\
\text { (Anxiogenic) }\end{array}$ \\
\hline & $\begin{array}{c}\text { Critchley } \\
\text { et al. }(1992)^{2}\end{array}$ & $\begin{array}{c}\text { Single } \\
(0.1-5, \text { IP })\end{array}$ & Not tested & Not tested & $\begin{array}{c}\text { Decreased } \\
\text { (Anxiogenic) }\end{array}$ \\
\hline & $\begin{array}{c}\text { Collinson and } \\
\text { Dawson (1997) }\end{array}$ & $\begin{array}{c}\text { Single } \\
(0.3-4, \text { IP })\end{array}$ & Not tested & Not tested & $\begin{array}{c}\text { Decreased } \\
\text { (Anxiolytic) }\end{array}$ \\
\hline & $\begin{array}{c}\text { Griebel } \\
\text { et al. (1997) }\end{array}$ & $\begin{array}{c}\text { Single } \\
(0.1-1, \mathrm{SC})\end{array}$ & Not tested & Not tested & $\begin{array}{l}\text { Increased }^{1} \\
\text { (Anxiolytic) }^{-}\end{array}$ \\
\hline \multirow{3}{*}{$\begin{array}{c}\text { Isapirone } \\
5-H T_{1 A} \\
\text { partial } \\
\text { agonist }\end{array}$} & $\begin{array}{c}\text { Graeff } \\
\text { et al. (1998) }\end{array}$ & $\begin{array}{c}\text { Single } \\
(0.25-2, \text { IP })\end{array}$ & $\begin{array}{l}\text { Impaired }^{1} \\
\text { (Anxiolytic) }\end{array}$ & Unchanged & Not tested \\
\hline & $\begin{array}{c}\text { Critchley } \\
\text { et al. }(1992)^{2}\end{array}$ & $\begin{array}{c}\text { Single } \\
(0.25-5, \text { IP })\end{array}$ & Not tested & Not tested & $\begin{array}{l}\text { Increased }^{1} \\
\text { (Anxiolytic) }\end{array}$ \\
\hline & $\begin{array}{c}\text { Setem } \\
\text { et al. (1999) }\end{array}$ & $\begin{array}{c}\text { Single } \\
(0.25-2.25)\end{array}$ & Not tested & Not tested & Unchanged \\
\hline \multirow{5}{*}{$\begin{array}{c}\text { Gepirone } \\
5-H T_{1 A} \\
\text { partial } \\
\text { agonist }\end{array}$} & $\begin{array}{c}\text { Critchley } \\
\text { et al. }(1992)^{2}\end{array}$ & $\begin{array}{c}\text { Single } \\
(0.1-5, \text { IP })\end{array}$ & Not tested & Not tested & Unchanged \\
\hline & $\begin{array}{c}\text { Motta } \\
\text { et al. (1992) }\end{array}$ & $\begin{array}{c}\text { Single } \\
(1-10, \mathrm{IP})\end{array}$ & Not tested & Not tested & $\begin{array}{c}\text { Decreased } \\
\text { (Anxiogenic) }\end{array}$ \\
\hline & & $\begin{array}{c}14 \text { days } \\
(1-10, \text { IP })\end{array}$ & Not tested & Not tested & $\begin{array}{l}\text { Increased }^{1} \\
\text { (Anxiolytic) }^{2}\end{array}$ \\
\hline & $\begin{array}{l}\text { Silva and } \\
\text { Brandão } \\
(2000)\end{array}$ & $\begin{array}{c}\text { Single } \\
(1-10, \text { IP) }\end{array}$ & Not tested & Not tested & $\begin{array}{c}\text { Decreased } \\
\text { (Anxiogenic) }\end{array}$ \\
\hline & & $\begin{array}{l}14 \text { days } \\
(10, \text { IP })\end{array}$ & Not tested & Not tested & $\begin{array}{l}\text { Increased }^{1} \\
\text { (Anxiolytic) }\end{array}$ \\
\hline
\end{tabular}


TABLE II (continuation)

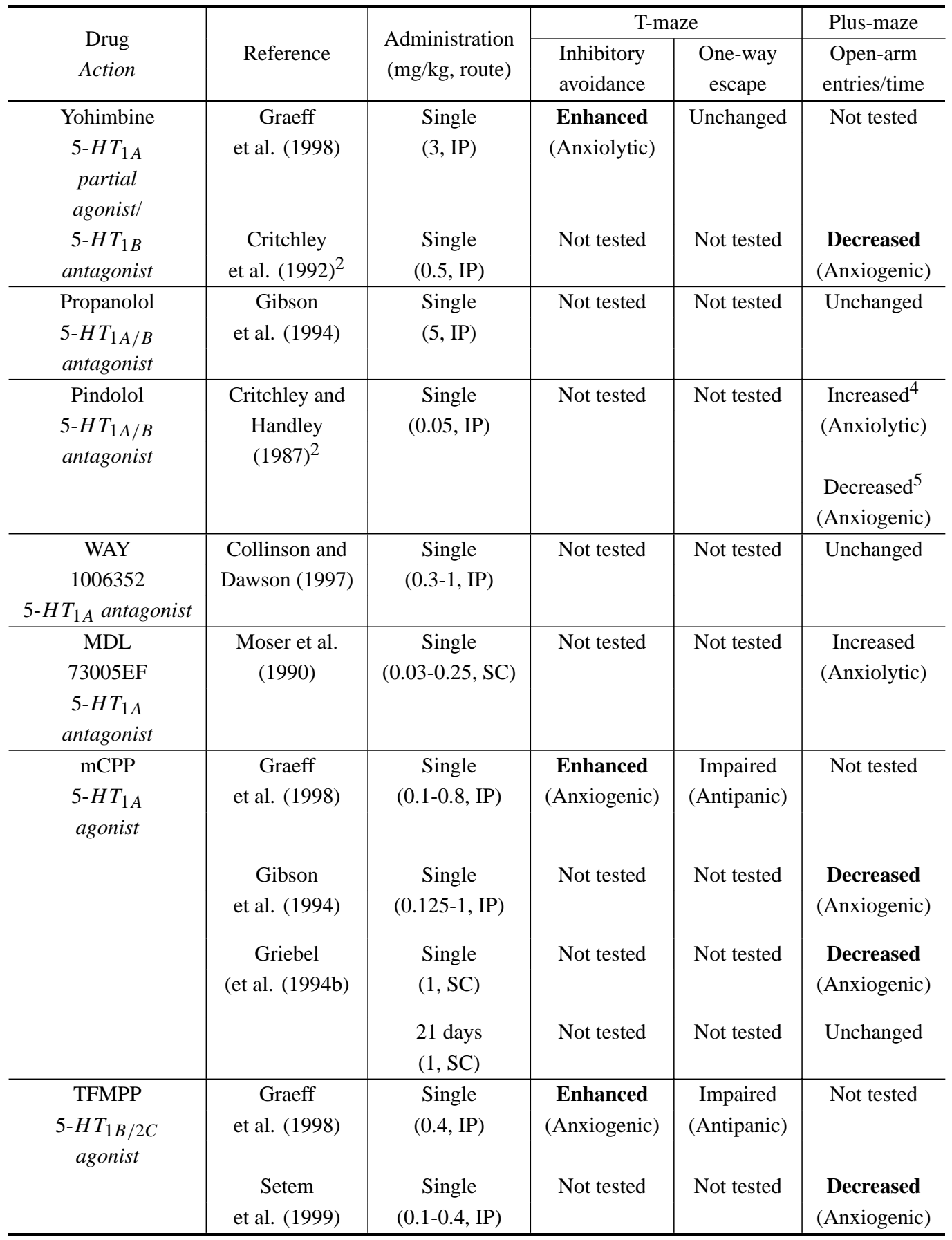


SIMONE H. PINHEIRO et al.

TABLE II (continuation)

\begin{tabular}{|c|c|c|c|c|c|}
\hline \multirow[b]{2}{*}{$\begin{array}{l}\text { Drug } \\
\text { Action }\end{array}$} & \multirow[b]{2}{*}{ Reference } & \multirow[b]{2}{*}{$\begin{array}{l}\text { Administration } \\
\text { (mg/kg, route) }\end{array}$} & \multicolumn{2}{|c|}{ T-maze } & \multirow{2}{*}{$\begin{array}{c}\text { Plus-maze } \\
\text { Open-arm } \\
\text { entries/time }\end{array}$} \\
\hline & & & $\begin{array}{l}\text { Inhibitory } \\
\text { avoidance }\end{array}$ & $\begin{array}{c}\text { One-way } \\
\text { escape }\end{array}$ & \\
\hline $\begin{array}{l}\text { Mianserin } \\
5-H T_{2 A} / C \\
\text { antagonist }\end{array}$ & $\begin{array}{c}\text { Griebel } \\
\text { et al. (1997) }\end{array}$ & $\begin{array}{c}\text { Single } \\
(0.3-3, \mathrm{SC})\end{array}$ & Not tested & Not tested & $\begin{array}{c}\text { Increased } \\
\text { (Anxiolytic) }\end{array}$ \\
\hline $\begin{array}{l}\text { Ritanserin } \\
5-H T_{2 A} / C \\
\text { antagonist }\end{array}$ & $\begin{array}{c}\text { Critchley and } \\
\text { Handley } \\
(1987)^{2}\end{array}$ & $\begin{array}{c}\text { Single } \\
(0.025-5, \mathrm{IP})\end{array}$ & Not tested & Not tested & $\begin{array}{l}\text { Increased }^{1} \\
\text { (Anxiolytic) }\end{array}$ \\
\hline $\begin{array}{l}\text { Ketanserin } \\
5-H T_{2 A} / C \\
\text { antagonist }\end{array}$ & $\begin{array}{c}\text { Critchley and } \\
\text { Handley } \\
(1987)^{2} \\
\text { Griebel } \\
\text { et al. (1997) } \\
\text { Motta } \\
\text { et al. (1992) }\end{array}$ & $\begin{array}{c}\text { Single } \\
(0.1-1, \mathrm{IP}) \\
\\
\text { Single } \\
(0.1-1, \mathrm{SC}) \\
\text { Single } \\
(0.5,1, \mathrm{IP})\end{array}$ & $\begin{array}{l}\text { Not tested } \\
\text { Not tested } \\
\text { Not tested }\end{array}$ & $\begin{array}{l}\text { Not tested } \\
\text { Not tested } \\
\text { Not tested }\end{array}$ & $\begin{array}{c}\text { Increased } \\
\text { (Anxiolytic) } \\
\text { Unchanged } \\
\text { Increased } \\
\text { (Anxiolytic) } \\
\text { Decreased } \\
\text { (Anxiogenic) }\end{array}$ \\
\hline $\begin{array}{l}\text { Seganserin } \\
5-H T_{2 A} / C \\
\text { antagonist }\end{array}$ & $\begin{array}{c}\text { Critchley and } \\
\text { Handley } \\
(1987)^{2}\end{array}$ & $\begin{array}{c}\text { Single } \\
(0.1-1, \text { IP })\end{array}$ & Not tested & Not tested & $\begin{array}{c}\text { Increased } \\
\text { (Anxiolytic) }\end{array}$ \\
\hline $\begin{array}{c}\text { Pirenperone } \\
5-H T_{2 A} / C \\
\text { antagonist }\end{array}$ & $\begin{array}{c}\text { Griebel } \\
\text { et al. (1997) }\end{array}$ & $\begin{array}{c}\text { Single } \\
(0.01-0.1, \mathrm{SC})\end{array}$ & Not tested & Not tested & $\begin{array}{c}\text { Decreased } \\
\text { (Anxiolytic) }\end{array}$ \\
\hline $\begin{array}{c}\text { SR 46349B } \\
\text { 5-HT } 2 A \\
\text { antagonist }\end{array}$ & $\begin{array}{c}\text { Graeff } \\
\text { et al. (1998) }\end{array}$ & $\begin{array}{c}\text { Single } \\
(1-10, \text { IP })\end{array}$ & $\begin{array}{c}\text { Impaired } \\
\text { (Anxiolytic) }\end{array}$ & Unchanged & Not tested \\
\hline $\begin{array}{c}\text { RP } 62203 \\
5-H T_{2 A} \\
\text { antagonist }\end{array}$ & $\begin{array}{c}\text { Graeff } \\
\text { et al. (1998) }\end{array}$ & $\begin{array}{c}\text { Single } \\
(0.25-4, \mathrm{IP})\end{array}$ & Unchanged & Unchanged & Not tested \\
\hline $\begin{array}{l}\text { SB } 200646 \mathrm{~A} \\
5-H T_{2 B} / C \\
\text { antagonist }\end{array}$ & $\begin{array}{c}\text { Graeff } \\
\text { et al. (1998) }\end{array}$ & $\begin{array}{c}\text { Single } \\
(3-30, \text { IP })\end{array}$ & $\begin{array}{c}\text { Impaired } \\
\text { (Anxiolytic) }\end{array}$ & Unchanged & Not tested \\
\hline $\begin{array}{c}\text { SER 082 } \\
\text { 5- } H T_{2 B / C} \\
\text { antagonist }\end{array}$ & $\begin{array}{c}\text { Graeff } \\
\text { et al. (1998) }\end{array}$ & $\begin{array}{c}\text { Single } \\
(0.1-1, \text { IP })\end{array}$ & $\begin{array}{c}\text { Impaired } \\
\text { (Anxiolytic) }\end{array}$ & Unchanged & Not tested \\
\hline $\begin{array}{c}\text { SB-242084 } \\
\text { 5-HT } 2 C \\
\text { antagonist }\end{array}$ & $\begin{array}{c}\text { Martin } \\
\text { et al. }(2002)\end{array}$ & $\begin{array}{c}\text { Single } \\
(0.1-3, \text { IP })\end{array}$ & Not tested & Not tested & $\begin{array}{c}\text { Increased } \\
\text { (Anxiolytic) }\end{array}$ \\
\hline
\end{tabular}

ministration and an anxiolytic effect following repeated administration for three weeks (Motta et al. 1992, Silva and Brandão 2000). One reported study in the X-maze has failed to show any effect of gepirone on the spatio temporal indexes of anxiety (Critchley et al. 1992). Like buspirone and isapirone, the clinical anxiolytic effect of gepirone becomes patent only after chronic administration. Therefore, the results reported in plus-maze bear at 
TABLE II (continuation)

\begin{tabular}{|c|c|c|c|c|c|}
\hline \multirow{2}{*}{$\begin{array}{l}\text { Drug } \\
\text { Action }\end{array}$} & \multirow[b]{2}{*}{ Reference } & \multirow{2}{*}{$\begin{array}{l}\text { Administration } \\
(\mathrm{mg} / \mathrm{kg}, \text { route) }\end{array}$} & \multicolumn{2}{|c|}{ T-maze } & \multirow{2}{*}{$\begin{array}{r}\text { Plus-maze } \\
\text { Open-arm } \\
\text { entries/time }\end{array}$} \\
\hline & & & $\begin{array}{l}\text { Inhibitory } \\
\text { avoidance }\end{array}$ & $\begin{array}{c}\text { One-way } \\
\text { escape }\end{array}$ & \\
\hline $\begin{array}{c}\text { Ondansetron } \\
\text { Zacopride } \\
\text { ICS } \\
\text { 205-9307 } \\
\text { MDL } \\
72222 \\
5-H_{3} \\
\text { antagonists }\end{array}$ & $\begin{array}{c}\text { Griebel } \\
\text { et al. (1997) }\end{array}$ & $\begin{array}{c}\text { Single } \\
(0.01-0.1, \mathrm{SC})\end{array}$ & Not tested & Not tested & Unchanged \\
\hline $\begin{array}{c}\text { BRL } \\
4670 \\
\text { 5-HT3 } \\
\text { antagonist }\end{array}$ & $\begin{array}{c}\text { Setem } \\
\text { et al. (1999) }\end{array}$ & $\begin{array}{c}\text { Single } \\
(0.001-0.1, \mathrm{IP})\end{array}$ & Not tested & Not tested & Unchanged \\
\hline
\end{tabular}

IP: intraperitoneal; SC: subcutaneous. ${ }^{1}$ Clinical improvement only after several weeks; ${ }^{2} \mathrm{X}$-maze; ${ }^{3}$ abolished by restraint for $1 \mathrm{~h}$ immediately before the test; ${ }^{4} 0.05-0.5 ;{ }^{5} 1 \mathrm{mg} / \mathrm{kg}$.

least partial correlation with the clinical evidence.

Overall, the results with the above three $5-\mathrm{HT}_{1 \mathrm{~A}}$ partial agonists suggest that the elevated T-maze can detect an anxiolytic effect of single administration. Although this does not strictly correlate with the time course of their clinical action, it may be useful for the laboratory screening of new anxiolytic agents.

With the exception of piremperone (Griebel et al. 1997) and of the $1 \mathrm{mg} / \mathrm{kg}$ dose of ketanserin (Motta et al. 1992), which enhanced anxiety in the elevated plusmaze, both selective and mixed antagonists of the $5-\mathrm{HT}_{2 \mathrm{~A}}$ and $5-\mathrm{HT}_{2 \mathrm{C}}$ receptor subtypes had anxiolytic effects either in the avoidance task of the T-maze or in the spatio temporal measures of the $\mathrm{X}$ or the plus maze. However, a direct comparison among mazes is prevented by the fact that each of these compounds has been tested in only one type of maze. Among the drugs studied, only ritanserin has been used in clinical assays, limiting the assessment of clinical correlations. In any case, the correlation found for ritanserin is positive, since this drug has been reported to improve GAD, although the therapeutic effect became significant only after three weeks of repeated administration (Ceulemans et al. 1985). Mianserin has been used clinically in depressed patients, but we have found no study focused on anxiety disorders. In addition, the ac- tions of mianserin are not restricted to 5-HT mechanisms, since this drug behaves as an antagonist on $\alpha_{2}$-adrenergic receptors.

The compounds designed in Table II by character and figure codes have been developed by drug companies as putative anxiolytic agents. With the exception of RP 62203 (Graeff et al. 1998), all of them had anxiolytic effects in the three elevated mazes, highlighting the usefulness of these animal models for screening new therapeutic agents. In addition, the consistent results reported with $5-\mathrm{HT}_{2 \mathrm{~A}}$ and $5-\mathrm{HT}_{2 \mathrm{C}}$ receptor antagonists support an important role played by these subtypes of 5-HT receptors in the neurobiology of anxiety (Wood 2003, Cohen 2005).

Three agents that increase anxiety in normal humans and/or aggravate symptoms of different anxiety disorders, namely the alkaloid yohimbine and the synthetic drugs $\mathrm{MCPP}$ and TFMPP, have been investigated in at least one of the elevated mazes. The mode of action of yohimbine is complex, not only because it affects more than one subtype of 5-HT receptors, but also because it behaves as an antagonist on $\alpha_{2}$-adrenergic receptors. In turn, mCPP and TFMPP act as preferential agonists on the $5-\mathrm{HT}_{2 \mathrm{C}}$ receptor. In agreement with their effect in human beings, the three agents facilitated the avoidance 
task in the T-maze (Graeff et al. 1998), and increased anxiety indexes in the plus-maze (Gibson et al. 1994, Griebel et al. 1994b) or in the X-maze (Critchley et al. 1992) after single administration. Interestingly, the anxiogenic effect of $\mathrm{mCPP}$ has no longer been detected after 21 days of repeated administration, indicating the development of tolerance (Griebel et al. 1994b).

The remaining 5-HT receptor agonists studied have not yet been investigated in human beings, so that correlation with human anxiety cannot be made. Among these drugs, the selective 5- $\mathrm{HT}_{1 \mathrm{~A}}$ receptor agonist 8-OH-DPAT has been the most frequently explored. The majority of the results obtained in either the $\mathrm{X}$ or the plus-maze show an anxiogenic effect. This is somewhat puzzling, since experimental evidence indicates that stimulation of 5-HT $\mathrm{H}_{1 \mathrm{~A}}$ receptors in forebrain limbic structures leads to anxiolytic effects (e.g., Zangrossi et al. 1999). An anxiolytic role of this receptor subtype is strongly supported by recent genetic evidence showing that mice devoid of $5-\mathrm{HT}_{1 \mathrm{~A}}$ receptors (knockout mice) look overanxious in several laboratory tests. Anxiety in mice is defined as a high level of avoidance of novel and unfamiliar environment and an increased fear reaction. Other aspects of anxiety such as autonomic activation, increased stress responsiveness, and neuroendocrine abnormalities have also been described in receptor knockout mice (Toth 2003).

A possible explanation is that systemically administered 8-OH-DPAT preferentially acts on pre-synaptic $5-\mathrm{HT}_{1 \mathrm{~A}}$ receptors localized in the cell bodies of serotonergic neurons, the stimulation of which results in decrease of neuronal firing and of the resulting 5-HT release from 5-HT terminals (Adell et al. 2002). In this way, post-synaptic $5-\mathrm{HT}_{1 \mathrm{~A}}$ receptors would actually be less stimulated than normally following systemic injection of 8-OH-DPAT. Supporting this hypothesis, opposite effects on flight induced by PAG stimulation have been reported by intracerebral administration of $8-\mathrm{OH}-$ DPAT either into the dorsal raphe nucleus or into the PAG, the former reproducing the effect of its systemic administration (Beckett and Marsden 1997). Partial 5-HT $1 \mathrm{~A}$ agonists, such as buspirone, have also been shown to reduce 5-HT neuron firing initially, but tolerance gradually develops along three weeks of repeated administration, testifying for the desensitization of pre-synaptic $5-\mathrm{HT}_{1 \mathrm{~A}}$ receptors (Blier and de Montigny 1990). It has even been suggested that this phenomenon would explain the delayed clinical action of the drug, which would be due, at least in part, to the overstimulation of post-synaptic 5- $\mathrm{HT}_{1 \mathrm{~A}}$ receptors (Mongeau et al. 1997).

\section{CONCLUSION}

From the evidence reviewed above, a comparative account among the mazes can be made.

The advantages of the elevated T-maze over the elevated $\mathrm{X}$ and plus mazes are:

1. The T-maze measures two behavioral tasks, namely inhibitory avoidance and one-way escape that represent two types of defense, respectively related to the emotions of anxiety and fear (panic).

2. There is good correlation between the effects of 5-HT reuptake inhibitors on the avoidance task and on GAD, as well as between those on the escape task and on PD.

3. Like the spatio temporal indexes of anxiety in the $\mathrm{X}$ and plus mazes, the avoidance task in the T-maze is sensitive to anxiolytic and anxiogenic drugs that act on 5-HT receptors, but the results obtained in the T-maze tend to be more consistent than in the other mazes.

The disadvantages are:

1. TFMPP and mCPP had an antipanic effect in the avoidance task, which is negatively correlated with the panicogenic action of these compounds that has been evidenced in clinical assays (Graeff et al. 2005).

2. An independent assessment of motor activity is necessary whenever the changes in avoidance and escape latency are in the same direction.

3. The tasks performed in the elevated T-maze cannot be automated, in contrast to the exploratory behavior displayed on the $\mathrm{X}$ and plus mazes.

It may be concluded that in terms of predictive value for drug response, the elevated T-maze is a promising model of GAD and PD. In addition, the reported results on the effects of intracerebral injection serotonergic 
drugs measured in the two tasks of the elevated T-maze support the proposal by Deakin and Graeff (1991) that 5-HT enhances anxiety by acting on forebrain limbic structures, whereas it inhibits panic by acting on the midbrain periaqueductal gray matter (for a recent review of the empirical evidence, see Graeff 2004). This highlights the usefulness of the elevated T-maze for testing hypothesis on the role of 5-HT in the pathophysiology of these disorders. Therefore, the model may also have some degree of theoretical or construct validity.

\section{ACKNOWLEDGMENTS}

This work was supported by a grant from Fundação de Amparo à Pesquisa do Estado de São Paulo (FAPESP) (2002/13197-2). FGG is recipient of research fellowships from Conselho Nacional de Desenvolvimento Científico e Tecnológico (CNPq) and Fundação de Apoio ao Ensino, Pesquisa e Assistência (FAEPA) do Hospital das Clínicas da Faculdade de Medicina de Ribeirão Preto, Universidade de São Paulo (FMRPUSP). We are indebted to Heloísa E.G. de Oliveira Graeff for the revision of English writing.

\section{RESUMO}

No presente artigo, revisamos resultados publicados relatando efeitos de drogas que atuam na neurotransmissão serotonérgica medidos em três labirintos elevados, que são modelos animais de ansiedade. Realizamos uma busca bibliográfica no MEDLINE, usando diferentes combinações das palavras-chave: $X$ maze, plus-maze, T-maze, serotonin e 5-HT, presentes no título ou no resumo, sem limite de tempo. Dos resumos obtidos, vários foram excluídos com base nos seguintes critérios: artigos de revisão que não continham resultados originais, espécies diferentes do rato, apenas injeções intracerebrais, ratos geneticamente manipulados, animais com algum tipo de patologia experimental. Os resultados relatados indicam que o efeito de drogas na tarefa de esquiva inibitória desempenhada no labirinto em T elevado, bem como nos índices espaciais de ansiedade nos labirintos em $\mathrm{X}$ ou em forma de cruz se correlacionam com os efeitos em pacientes diagnosticados com o transtorno de ansiedade generalizada. Por outro lado, os efeitos de drogas na tarefa de fuga unidirecional do labirinto em $\mathrm{T}$ predizem a resposta a drogas dos pacientes com o transtorno de pânico. De modo geral, os efeitos de drogas sobre a tarefa de esquiva no labirinto em $\mathrm{T}$ são mais consistentes que os medidos pe- los índices de ansiedade calculados nos labirintos em $\mathrm{X}$ e em forma de cruz. Portanto, o labirinto em T-elevado é um modelo promissor dos transtornos de ansiedade generalizada e de pânico.

Palavras-chave: modelo animal, labirinto elevado, ansiedade, pânico, serotonina.

\section{REFERENCES}

Adell A, Celada P, Abellan MT and Artigas F. 2002. Origin and functional role of the extracellular serotonin in the midbrain raphe nuclei. Brain Res Rev 39: 154-180.

American Psychiatric Association. 1994. Diagnostic and statistical manual of mental disorders, $4^{\text {th }}$ ed., American Psychiatric Association Press, Washington, DC.

Ballenger JC, Wheadon DE, Steiner M, Bushnell W AND Gergel IP. 1998. Double-blind, fixed-dose, placebo-controlled study of paroxetine in the treatment of panic disorder. 155: 36-42.

Beckett S And MARSDEN CA. 1997. The effect of central and systemic injection of the $5-\mathrm{HT}_{1 \mathrm{~A}}$ receptor agonist 8 -OHDPAT and the $5-\mathrm{HT}_{1 \mathrm{~A}}$ receptors antagonist WAY 100635 on periaqueductal grey-induced defence behaviour. J Psychopharmacol 11: 35-40.

BEIJAMini V AND ANDREATINI R. 2003. Effects of Hypericum perforatum and paroxetine on rat performance in the elevated T-maze. Pharmacol Res 48: 199-207.

BLier P AND De Montigny C. 1990. Electrophysiological investigation of the adaptive response of the 5-HT system to the administration of $5-\mathrm{HT}_{1 \mathrm{~A}}$ receptor agonists. J Cardiovasc Pharmacol 15 (Suppl 7): S42-S48.

Ceulemans Dls, Hoppenbrouwers MlJa, Gelders YG AND REYNTJENS AJM. 1985. The influence of ritanserin, a serotonin antagonist in anxiety disorders: A double-blind placebo-controlled study versus lorazepam. Pharmacopsychiatry 18: 303-305.

CoHen H. 2005. Anxiolytic effect and memory improvement in rats by antisense oligodeoxynucleotide to 5-hydroxytryptamine-2A precursor protein. Depress Anxiety 22: 84-93.

Collinson N And DAwson GR. 1997. On the elevated plus-maze the anxiolytic-like effects of the 5-HT(1A) agonist, 8-OH-DPAT, but not the anxiogenic-like effects of the 5-HT(1A) partial agonist, buspirone, are blocked by the 5-HT $\mathrm{HA}_{1 \mathrm{~A}}$ antagonist, WAY 100635. Psychopharmacology 132: 35-43. 
CRITChley MA AND HANDLEY SL. 1987. Effects in the Xmaze anxiety model of agents acting at $5-\mathrm{HT}_{1}$ and $5-\mathrm{HT}_{2}$ receptors. Psychopharmacology 93: 502-506.

CRitchley MA, NuUnge K AND HANDley SL. 1992. Actions and some interactions of 5- $\mathrm{HT}_{1 \mathrm{~A}}$ ligands in the elevated X-maze and effects of dorsal raphe lesions. Psychopharmacology 106: 484-490.

CRUZ APM, Frei F AND GRAeFF FG. 1994. Ethopharmacological analysis of rat behavior on the elevated plusmaze. Pharmacol Biochem Behav 49: 171-176.

Cutler NR, Sramek JJ, Keppel Hesslink JM, Krol A, Roeschen J, Rockels K AND SchWeIzer E. 1993. A double-blind, placebo-controlled study comparing the efficacy and safety of ipsapirone versus lorazepam in patients with generalized anxiety disorder: a prospective multicenter trial. J Clin Psychopharmacol 13: 429 437.

DEAKIN JFW AND GRAEFF FG. 1991. 5-HT and mechanisms of defence. J Psychopharmacol 5: 305-315.

Estes WK AND SKINNER FB. 1941. Some quantitative properties of anxiety. J Exp Psychol 29: 390-400.

Gentil V, Lotufo-Neto F, Andrade L, Cordás T, Bernik M, Ramos R, Maciel L, Miyakawa E AND GoRensteIn C. 1993. Clomipramine, a better reference drug for panic/agoraphobia. I. Effectiveness comparison with imipramine. J Psychopharmacol 7: 316-324.

GiBSON EL, BARNFILD AM AND CURZON G. 1994. Evidence that $\mathrm{mCPP}$-induced anxiety in the plus-maze is mediated by postsynaptic $5-\mathrm{HT}_{2 \mathrm{C}}$ receptors but not by sympathomimetic effects. Neuropharmacology 33: 457-465.

GRAEFF FG. 2004. Serotonin, the periaqueductal gray and panic disorder. Neurosci Biobehav Rev 28: 239-259.

GraefF FG, VianA MB And Tomaz C. 1993. The elevated T maze, a new experimental model of anxiety and memory: Effect of diazepam. Braz J Med Biol Res 26: 67-70.

GraefF FG, Viana MB, Mora PO. 1996. Opposed regulation by dorsal raphe nucleus 5-HT pathways of two types of fear in the elevated T-maze. Pharmacol Biochem Behav. 53: 171-177.

GraefF FG, Netto CF And Zangrossi-JR H. 1998. The elevated T-maze as an experimental model of anxiety. Neurosci Biobehav Rev 23: 237-246.

Graeff FG, Garcia-Leal C, Del-Ben CM, GuimaRÃES FS. 2005. Does the panic attack activate the hypothalamic-pituitary-adrenal axis? An Acad Bras Cienc 77: $1-15$.
GRIEBEL G. 1995. 5-Hydroxytryptamine-interacting drugs in animal models of anxiety disorders: more than 30 years of research. Pharmacol Ther 65: 319-395.

Griebel G, Moreau JL, Jenck F, Misslin R and MarTIN JR. 1994a. Acute and chronic treatment with 5-HT reuptake inhibitors differentially modulate emotional responses in anxiety models in rodents. Psychopharmacology 113: 463-470.

Griebel G, Moreau JL, Jenck F, Mutel V, Martin JR AND MISSLIN R. 1994b. Evidence that tolerance to the anxiogenic-like effects of mCPP does not involve alteration in the function of 5-HT(2C) receptors in the rat choroid plexus. Behav Pharmacol 5: 642-645.

Griebel G, Rodgers RJ, Perrault G and Sanger DJ. 1997. Risk assessment behaviour: evaluation of utility in the study of 5-HT-related drugs in the rat elevated plusmaze test. Pharmacol Biochem Behav 57: 817-827.

Griebel G, Cohen C, Perrault G and Sanger DJ. 1999. Behavioral effects of acute and chronic fluoxetine in Wistar-Kyoto rats. Physiol Behav 67: 315-320.

HANDlEy SL AND Mithani S. 1984. Effects of alphaadrenoceptor agonists in a maze-exploration model of "fear"-motivated behaviour, Archiv Pharmacol 327: 1-5.

HANDley SL, McBlane JW, CRITChley MAE AND NJUNGE K. 1993. Multiple serotonin mechanisms in animal models of anxiety: environmental, emotional and cognitive factors. Behav Brain Res 58: 203-210.

Jorge SD, Pobre RL, De Paula SV, Oliveira AM AND ZANGROSSI-JR H. 2004. Effects of sibutramine on anxiety-related behaviours in rats. Pharmacol Res 50: 517-522.

KAHN RJ, MaCNAIR DM, Liman RS, Covi L, Rickels K, Downing R, Fischer S And GrakenthaleR LM. 1986. Imipramine and chlordiazepoxide in depressive and anxiety disorders. II. Efficacy in anxious outpatients. Arch Gen Psychiat 43: 79-85.

Kelleher RT ANd Morse WH. 1968. Determinants of the specificity of behavioral effects of drugs. Ergeb Physiol 60: $1-56$.

KLEIN DF. 1964. Delineation of two drug-responsive anxiety syndromes. Psychopharmacology 5: 397-408.

Koks S, Beljajev S, Koovit I, Abramov U, Bourin M AND VASAR E. 2001. 8-OH-DPAT, but not deramciclane, antagonizes the anxiogenic-like action of paroxetine in an elevated plus-maze. Psychopharmacology 153: $365-372$. 
Martin JR, BALlard TM, ANd Higgina GA. 2002. Influence of the $5-\mathrm{HT}_{2 \mathrm{C}}$ receptor antagonist, $\mathrm{SB}-242084$, in tests of anxiety. Pharmacol Biochem Behav 71: 615-625.

McBlane JW And Handley SL. 1994. Effects of two stressors on behavior in the elevated X-maze: preliminary investigation of their interaction with 8-OH-DPAT. Psychopharmacology 116: 173-182.

Millenson JR AND LeSLIE J. 1974. The conditioned emotional response (CER) as a baseline for the study of antianxiety drugs. Neuropharmacology 13: 1-9.

Mongeau R, Blier P and De Montigny C. 1997. The serotonergic and noradrenergic systems of the hippocampus: their interactions and the effects of antidepressant treatments. Brain Res Bull 23: 145-195.

Moser PC, Tricleblank MD, Middlemiss DN, Mir AK, Hibert MF AND FOZARD JR. 1990. Characterization of MDL $73005 \mathrm{EF}$ as a 5- $\mathrm{HT}_{1 \mathrm{~A}}$ selective ligand and its effects in animal models of anxiety: comparison with buspirone, 8-OH-DPAT and diazepam. Br J Pharmacol 99: 343-349.

Motta V, Maisonette S, Morato S, Castrechini P AND BRANDÃO ML. 1992. Effects of blockade of 5$\mathrm{HT}_{2}$ receptors and activation of $5-\mathrm{HT}_{1 \mathrm{~A}}$ receptors on the exploratory activity of rats in the elevated plus-maze. Psychopharmacology 107: 135-139.

NinAN PT AND DUNLOP BW. 2005. Neurobiology and etiology of panic disorder. J Clin Psychiatry 66 (Suppl 4): 3-7.

NUTT DJ. 2005. Overview of diagnosis and drug treatments of anxiety disorders. CNS Spectr 10: 49-56.

PECKNOLD JC. 1997. A risk-benefit assessment of buspirone in the treatment of anxiety disorders. Drug Saf 16: 118132.

Pellow S, Chopin P, File SE And Briley M. 1985. Validation of open:closed arm entries in the elevated plus-maze as a measure of anxiety in the rat. J Neurosci Meth 14: 149-67.

Poltronieri C, Zangrossi-Jr H ANd De Barros VIANA M. 2003. Antipanic-like effect of serotonin reuptake inhibitors in the elevated T-maze. Behav Brain Res 147: 185-192.

Rickels K, Schweizer E, Demartinis N, Mandos L AND MerCer C. 1997. Gepirone and diazepam in generalized anxiety disorder: a placebo-controlled trial. J Clin Psychopharmacol 7: 272-277.

RODGERS RJ AND JOHNSON NJT. 1995. Factor analysis of spatio temporal and ethological measures in the murine elevated plus-maze. Pharmacol Biochem Behav 49: $297-$ 303.

SCHWEIZER E, Rickels K, WeISS S ANd ZAVODNICK S. 1993. Maintenance drug treatment for panic disorder. I. Results of a prospective, placebo-controlled comparizon of alprazolam and imipramine. Arch Gen Psychiatry 50: 51-60.

Setem J, Pinheiro AP, Motta VA, Morato S And CRUZ AP. 1999. Ethopharmacological analysis of 5-HT ligands on the rat elevated plus-maze. Pharmacol Biochem Behav 62: 515-521.

Silva RC AND BRANDÃo ML. 2000. Acute and chronic effects of gepirone and fluoxetine in rats tested in the elevated plus-maze: an ethological analysis. Pharmacol Biochem Behav 65: 209-216.

Silva MT, Alves CR and Santarem EM. 1999. Anxiogenic-like effect of acute and chronic fluoxetine on rats tested on the elevated plus-maze. Braz J Med Biol Res 32: 333-339.

TEIXeIRA RC, ZANGRossi-JR H AND GRAEFF FG. 2000. Behavioral effects of acute and chronic imipramine in the elevated T-maze model of anxiety. Pharmacol Biochem Behav 65: 571-576.

Tотн M. 2003. 5-HT $1 \mathrm{~A}$ receptor knockout mouse as a genetic model of anxiety. Eur J Pharmacol 463: 177-184.

TREIT D. 1985. Animal models for the study of anti-anxiety agents: A review. Neurosci Biobehav Rev 9: 203-222.

Wood MD. 2003. Therapeutic potential of 5-HT $2 \mathrm{C}$ receptor antagonists in the treatment of anxiety disorders. Curr Drug Targets CNS Neurol Disord 2: 383-387.

ZANGROSSI-JR H AND GRAEFF FG. 1997. Behavioral validation of the elevated T-maze, a new animal model of anxiety. Brain Research Bulletin 44: 1-5.

ZANGROSSI-JR H, ViAnA MB AND GRAEFF FG. 1999. Anxiolytic effect of intra-amygdala injection of midazolam and 8-hydroxy-2(di-n-propylamino)tetralin in the elevated T-maze. Eur J Pharmacol 369: 267-270.

Zanoveli JM, Netto CF, Guimarães FS AND ZANGROSSI-JR H. 2004. Systemic and intra-dorsal periaqueductal gray injections of cholecystokinin sulfated octapeptide (CCK-8s) induce a panic-like response in rats submitted to the elevated T-maze. Peptides 25: 1935-1941.

ZANOVEli JM, Nogueira RL AND ZANGROSSI-JR H. 2005. Chronic imipramine treatment sensitizes $5-\mathrm{HT}_{1 \mathrm{~A}}$ and $5-\mathrm{HT}_{2 \mathrm{~A}}$ receptors in the dorsal periaqueductal gray matter: evidence from the elevated T-maze test of anxiety. Behav Pharmacol 16: 543-552. 